\title{
Massive AGB models of low metallicity: the implications for the self-enrichment scenario in metal-poor globular clusters
}

\author{
P. Ventura and F. D’Antona
}

\author{
INAF - Observatory of Rome, via Frascati 33, 00040 MontePorzio Catone (RM), Italy \\ e-mail: [ventura; dantona]@oa-roma.inaf.it
}

Received 13 October 2008 / Accepted 16 February 2009

\section{ABSTRACT}

\begin{abstract}
Context. We present the physical and chemical properties of intermediate-mass star models of low metallicity, as they evolve along the thermal pulse phase.

Aims. We extend to low metallicities, of $Z=1,2$ and $6 \times 10^{-4}$, models previously computed for chemical compositions typical of globular clusters of an intermediate metallicity $(Z=0.001)$, and for the most metal-rich clusters found in our Galaxy $(Z=0.004)$. We aim to test in particular the self-enrichment scenario for metal-poor globular clusters.

Methods. We calculated three grids of intermediate-mass models with metallicities $Z=10^{-4}, 2 \times 10^{-4}$, and $6 \times 10^{-4}$, following their evolutionary sequences from the pre-main-sequence to the asymptotic giant branch phase, almost until the ejection of the entire envelope. We discuss the chemistry of the ejecta, and, in particular, the mass fractions of elements that have been studied in the numerous, deep, spectroscopic surveys of globular clusters.

Results. Although oxygen and sodium data are scarce for low-metallicity globular clusters, the small amonut of data avalilable for the unevolved stars in NGC 6397 are compatible with the models. Furthermore, we find good agreement with the C-N anticorrelation of unevolved stars in the cluster M 15. In this cluster, however, no stars of low oxygen $([\mathrm{O} / \mathrm{Fe}] \sim-1)$ abundance have been detected. The most massive, very metal-poor clusters, should contain such stars, according to the present models. At the lowest metallicity $Z=10^{-4}$, the ejecta of the most massive AGBs have $\mathrm{C} / \mathrm{O}>1$, due to the dramatic decrease in the oxygen abundance. We discuss the possible implications of this prediction.
\end{abstract}

Key words. stars: abundances - stars: AGB and post-AGB - stars: evolution - stars: chemically peculiar - globular clusters: general

\section{Introduction}

The evolution of massive asymptotic giant Branch stars (hereafter AGB, i.e., stars with masses $4 M_{\odot} \leq M \leq 7 M_{\odot}$ during the thermal pulse phase) has been the subject of extensive studies by several research groups, primarily because they have been proposed to be responsible for the differences in the surface chemistry of globular clusters (GCs) stars. GC stars exhibit both considerable differences in their surface abundances of the "light" elements $(A<30)$ (Kraft 1994), and clearly evident abundance patterns, involving all elements to aluminium (see Carretta 2006, for a review on this topic). The idea that these apparent anomalies were formed "in situ" by some noncanonical extra-mixing at the bottom of the convective envelope during red giant branch (RGB) evolution (Denissenkov \& Weiss 2001) was excluded by the discovery that even cool turnoff (TO) or subgiant branch (SGB) stars exhibited similar patterns (Gratton et al. 2001), indicating that the presently observed surface chemical compositions were identical to when the stars formed. This led to the development of "self-enrichment" scenarios, in which stars with anomalous chemical composition (second generation - hereinafter SG - stars) formed in an interstellar medium polluted by the winds of an earlier generation of stars (the first generation or FG). It has been proposed that the winds of either massive AGBs (Cottrell \& Da Costa 1981; Ventura et al. 2001) or rapidly rotating massive stars (Prantzos \& Charbonnel 2006) are the progenitors of the SG, the latter scenario being described in detail in Decressin et al. (2007) and references therein. Here, we follow the first hypothesis. The massive AGBs evolve at young ages ( 40-100 Myr) compared to the typical ages of GCs $(\sim 10-15 \mathrm{Gyr})$ and complete, at the bottom of their surface convective zone, an advanced stage of nucleosynthesis (Hot Bottom Burning, hereinafter HBB, Blöcker \& Schönberner 1991; D'Antona \& Mazzitelli 1996), the products of which should be carried to the surface by convective currents (Ventura et al. 2001). The medium in which this generation of stars evolved should thus show the signature of this nucleosynthesis, because the low velocities $\left(\sim 10-20 \mathrm{~km} \mathrm{~s}^{-1}\right)$ of these winds allow them to be retained inside the globular cluster. If the initial mass function (IMF) of the FG is standard (e.g., a Salpeter 1955; or Kroupa et al. 1993, IMF) the gas contained in the massive AGB ejecta will be too scarce to explain the origin of a SG containing a large fraction of today's cluster stars shown from observation. Therefore, most of the FG stars have been proposed to be lost, as confirmed by some dynamical and $N$-body models (D'Ercole et al. 2008) or the GC may have collected the AGB gas from a far wider environment, e.g., if it was born in a dwarf galaxy that today is dispersed (Bekki \& Norris 2006; Renzini 2008). A robust prediction from stellar evolution models is that the ejecta from massive AGBs, such as those from massive stars (Smith 2006), are expected to be helium-rich. The existence of a helium-rich population $(Y>0.30)$ provides an appealing explanation for the existence of some GCs with Horizontal Branches (HBs) showing an extended blue tail (D'Antona et al. 2002). This idea has been elaborated in many subsequent works (e.g., D'Antona \& Caloi 2004; Lee et al. 2005; Caloi \& D'Antona 2005, 2007; Busso et al. 2007; D'Antona \& Caloi 2008). The presence of a fraction of stars enriched in helium has also been 
inferred by the presence of a blue main sequence in $\omega$ Cen (Bedin et al. 2004; Norris 2004; Piotto et al. 2005), and in NGC 2808 (Piotto et al. 2007).

However, the debate concerning the possible role that massive AGBs may have played in the self-enrichment scenario is still open, because AGB modeling proves to be one of the most delicate and uncertain tasks in the study of stellar evolution. This is the reason why results presented by the various groups working on this topic are sometimes extremely different, leading to opposite conclusions concerning many physical and chemical properties of the evolution of this class of objects (Denissenkov \& Herwig 2003; Fenner at al. 2004; Karakas \& Lattanzio 2007, KL07; Ventura et al. 2001).

Ventura \& D'Antona (2005a,b, 2006) showed that discrepancies in the results obtained by various investigators are a consequence of their different description of some physical phenomena. In more detail, Ventura \& D'Antona (2005a) showed that the efficiency of the convective model adopted may change substantially the evolution of the main physical properties of these stars, e.g., the duration of the entire AGB phase, the maximum luminosity reached, and the degree of nucleosynthesis achieved at the bottom of the convective envelope. The two main arguments against the self-enrichment scenario raised by some investigators are that the winds of even the most massive AGBs should exhibit only a modest extent of oxygen depletion and a great enhancement in the overall CNO abundances (Fenner at al. 2004), which are at odds with the observational evidence (Ivans et al. 1999), and are due entirely to the use of the Mixing Length Theory (MLT, Vitense 1953). When the Full Spectrum of Turbulence (FST, Canuto \& Mazzitelli 1991) approach is used to model convection, a much stronger HBB and a much smaller CNO enhancement is found (Ventura \& D'Antona 2005a).

The uncertainties associated with mass loss have a smaller impact on the results obtained, as extensively discussed in Ventura \& D'Antona (2005b).

Ventura \& D' Antona (2008a,b) presented their updated AGB models for testing the self-enrichment scenario for GCs of intermediate $(Z=0.001)$ and higher $(Z=0.004)$ metallicities. These works were a more systematic treatment of this issue, designed to integrate their qualitative approach discussed in D'Antona \& Ventura (2007), and limited only to the $Z=0.001$ case. The main results of these investigations were that the most massive FST AGB models $\left(M \sim 5-6 M_{\odot}\right)$ produce ejecta whose chemical composition agrees with the abundance patterns observed in TO and SGB stars in GCs, in terms of oxygen-sodium, oxygenaluminium, and carbon-nitrogen anticorrelations. These results require a choice of the nuclear cross-sections involving proton capture by ${ }^{22} \mathrm{Ne},{ }^{23} \mathrm{Na},{ }^{25} \mathrm{Mg}$, and ${ }^{26} \mathrm{Mg}$ nuclei, within the range allowed by the uncertainties (Hale et al. 2002, 2004). The most extreme anomalies, observed only in low gravity giants, can be explained possibly by non-canonical extra-mixing during the RGB phase, which would be consistent with the lower height of the entropy barrier at the hydrogen-helium interface expected in the stars belonging to the SG (D'Antona \& Ventura 2007).

We extend the results of the above mentioned investigations to lower metallicities. With this intention, we calculated new evolutionary sequences for models of intermediate mass with metallicities $Z=10^{-4}, 2 \times 10^{-4}, 6 \times 10^{-4}$. These computations complete the theoretical framework concerning massive AGBs of chemistry typical of GC stars, and allow us to test the validity of the self-enrichment scenario for the most metal-poor GCs, comparing the chemistry of the theoretical ejecta of these models with the abundance patterns shown by the spectroscopic investigations of the GCs M 15 (Sneden et al. 1997; Cohen et al. 2005) and NGC 6397 (Carretta et al. 2005).

The paper is organized as follows. Section 2 describes the physical and chemical ingredients used to calculate the evolutionary sequences. The physical properties of the models are presented in Sect. 3. The chemical content of their ejecta, and its dependence on mass and metallicity, is discussed in Sect. 4, and compared with observations in Sect. 5.

\section{The physical and chemical inputs}

All evolutions presented in this work were calculated with the ATON code for stellar evolution, and the numerical structure described in detail in Ventura et al. (1998). We adopt the latest opacities by Ferguson et al. (2005) at temperatures lower than $10000 \mathrm{~K}$ and the OPAL opacities in the version documented by Iglesias \& Rogers (1996). The mixture adopted is alphaenhanced with $[\alpha / \mathrm{Fe}]=0.4$ (Grevesse \& Sauval 1998). The conductive opacities were taken from Poteckhin (2006) ${ }^{1}$, and are added harmonically to the radiative opacities. Tables of the equation of state are generated in the (gas) pressure-temperature plane, according to the latest release of the OPAL EOS (2005), overwritten in the pressure ionization regime by the EOS by Saumon et al. (1995), and extended to the high-density, hightemperature domain according to the treatment by Stoltzmann \& Blöcker (2000). Convection was modelled according to the FST prescription. Mixing of chemicals within convective zones was treated as a diffusive process. We followed the approach by Cloutman \& Eoll (1976), solving the diffusive-like equation for each chemical species:

$$
\frac{\mathrm{d} X_{i}}{\mathrm{~d} t}=\left(\frac{\partial X_{i}}{\partial t}\right)_{\text {nucl }}+\frac{\partial}{\partial m_{r}}\left[\left(4 \pi r^{2} \rho\right)^{2} D \frac{\partial X_{i}}{\partial m_{r}}\right]
$$

where $D$ is the diffusion coefficient, for which, given the convective velocity $v$ and the scale of mixing $l$, a local approximation $\left(D \sim \frac{1}{3} v l\right)$ was adopted. The borders of the convective regions were fixed according to the Schwarzschild criterium. We considered extra-mixing from all the formal convective boundaries to the beginning of the AGB phase: convective velocities were assumed to decay exponentially with an e-folding distance described by the free-parameter $\zeta$, which was set to be $\zeta=0.02$, according to the calibration provided in Ventura et al. (1998), where the interested reader can also find a complete discussion regarding the variation in the convective velocities within the proximity of the convective borders. No extra-mixing was assumed to occur for the remainder of the AGB phase: these results therefore provide a conservative estimate of the extent of the Third Dredge-up following each thermal pulse.

Mass loss was described according to the Blöcker (1995) formulation, which is more accurate than the basic Reimer's recipe in describing the steep increase in mass loss with luminosity as the stars climb the AGB in the HR diagram. The full expression is

$\dot{M}=4.83 \times 10^{-22} \eta_{\mathrm{R}} M^{-3.1} L^{3.7} R$,

where $\eta_{\mathrm{R}}$ is the free parameter entering the Reimers' prescription, for which we used $\eta_{\mathrm{R}}=0.02$, according to the calibration based on the luminosity function of lithium-rich stars in the Magellanic Clouds given by Ventura et al. (2000). The nuclear network includes 30 elements (up to ${ }^{31} \mathrm{P}$ ) and 64 reactions, a full list of which can be found in Ventura \& D'Antona (2005a). The relevant cross-sections are taken from the recommended values

\footnotetext{
1 See the web page www. ioffe.rssi.ru/astro/conduct/
} 


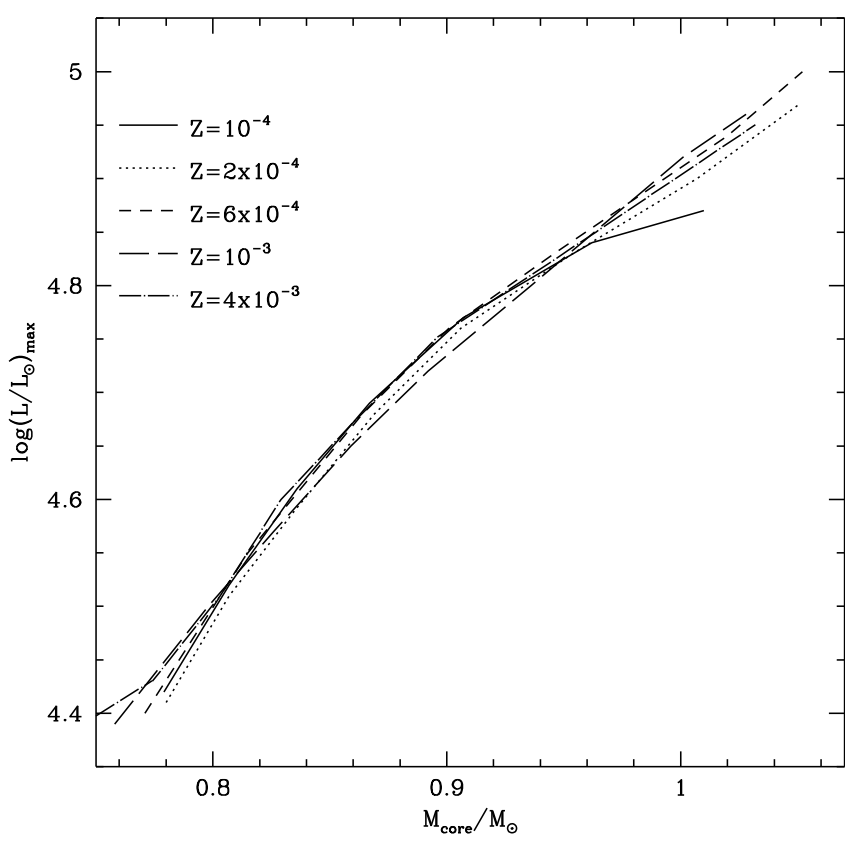

Fig. 1. Variation of the maximum luminosity attained by the AGB models as a function of the core mass at the beginning of the AGB phase, when the CNO shell is extinguished after the core He-burning phase.

in the NACRE compilation (Angulo et al. 1999) with only the following exceptions:

1. ${ }^{14} \mathrm{~N}(\mathrm{p}, \gamma){ }^{15} \mathrm{O}$ (Formicola et al. 2004);

2. ${ }^{22} \mathrm{Ne}(\mathrm{p}, \gamma){ }^{23} \mathrm{Na}$ (Hale et al. 2002);

3. ${ }^{23} \mathrm{Na}(\mathrm{p}, \gamma){ }^{24} \mathrm{Mg}$ (Hale et al. 2004);

4. ${ }^{23} \mathrm{Na}(\mathrm{p}, \alpha){ }^{20} \mathrm{Ne}$ (Hale et al. 2004);

5. ${ }^{25} \mathrm{Mg}(\mathrm{p}, \gamma){ }^{26} \mathrm{Al}$ (NACRE, upper limits);

6. ${ }^{26} \mathrm{Mg}(\mathrm{p}, \gamma){ }^{27} \mathrm{Al}$ (NACRE, upper limits).

\section{The physical properties of the low Z AGB models}

The models presented here were calculated assuming metallicities $Z=2 \times 10^{-4}$ and $Z=6 \times 10^{-4}$. The mixtures were assumed to be alpha-enhanced with $[\alpha / \mathrm{Fe}]=+0.4$, such that these two chemical compositions correspond to $[\mathrm{Fe} / \mathrm{H}]=-2.3$ and $[\mathrm{Fe} / \mathrm{H}]=-1.83$ respectively, encompassing the chemistry of the most metal-poor GCs. We also computed a set of models with metallicity $Z=10^{-4}$, to allow direct comparison with the metal-poor models of Karakas \& Lattanzio (2007) and Herwig (2004).

The main physical properties of the models for the various metallicities are reported in Table 1 . We also show the results by Ventura \& D'Antona (2008a) for $Z=10^{-3}$ and Ventura \& D'Antona (2008b) for $Z=4 \times 10^{-3}$. The columns in the table indicate the initial mass of the model, the duration of the two core nuclear-burning phases, the core mass at the beginning of the AGB phase (when the hydrogen shell is extinguished after the exhaustion of the central helium), the maximum luminosities and the maximum temperatures at the bottom of the convective envelope reached during the AGB phase, the number of thermal pulses experienced by the star before all the envelope is lost, and the maximum value of the third dredge-up (hereinafter TDU) parameter $\lambda$, defined to be the ratio of the mass dredged-up in the after-pulse phase to the increase in the core mass (due to the outward advance of the CNO burning shell) from the previous pulse.

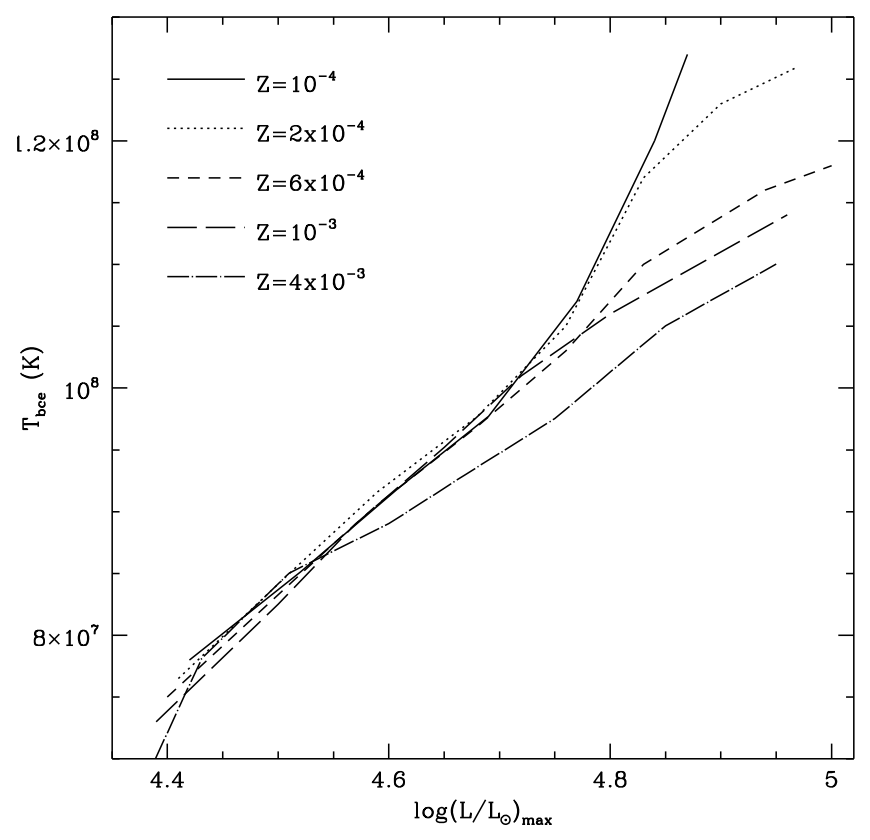

Fig. 2. The variation in the maximum temperature attained at the bottom of the convective envelope of the AGB models of various metallicities as a function of the maximum luminosity reached.

For a given initial mass $M$, we see from the 4th column of Table 1 that the core mass increases with decreasing $Z$, a wellknown result of stellar evolution theories; we note a small difference between the properties of the $Z=10^{-4}$ and $Z=2 \times 10^{-4}$ sets of models.

Figure 1 shows that, independently of the metallicity, during the AGB phase the models follow approximately the same relationship between the core mass at the beginning of the AGB phase $\left(M_{\mathrm{C}}\right)$ and the maximum luminosity reached $\left(L_{\max }\right)$ in almost all the range of $M_{\mathrm{C}} \mathrm{s}$ spanned by the models; Fig. 1 shows indeed a flattening of the $M_{\mathrm{C}}-L_{\max }$ trend at the lowest metallicities, whereas the higher $Z$ models follow a steeper behaviour. This result can be understood to be caused by the variation with $Z$ in the mass fraction of the chemical species involved in the $\mathrm{CNO}$ cycle, which becomes particularly relevant whenever strong HBB conditions are reached, for large M: the "extraluminosity" gained by the star as a consequence of the proximity of the bottom of the convective envelope to the CNO shell peak (Ventura \& D'Antona 2005a) increases with the mass fractions of the CNO elements, and hence with $Z$. This is also why, for masses close to the limit for carbon ignition $\left(\sim 6 M_{\odot}\right.$ in the present investigation), the common behaviour of low $Z$ models attaining higher luminosities for a given initial mass is reversed, as we can see from Cols. 1 and 3 of Table 1 ; the $6 M_{\odot}$ model of metallicity $Z=10^{-3}$ reaches a higher luminosity than its lower $Z$ counterparts, despite having a smaller core mass.

The main difference among the various sets of models presented here is the temperature at the bottom of the convective envelope: Fig. 2 shows that for a given luminosity the low $Z$ models are hotter. This behaviour is again a consequence of the smaller mass fractions of the CNO elements at lower $Z$, which requires a higher shell temperature to reach the same luminosity.

We conclude this analysis by discussing the number of thermal pulses (NTP) experienced by the various masses, shown as a function of the initial mass in Fig. 3. For a given mass $M$, NTP decreases with $Z$, as expected because higher $Z$ models have larger radii, and suffer a more significant mass loss, which 


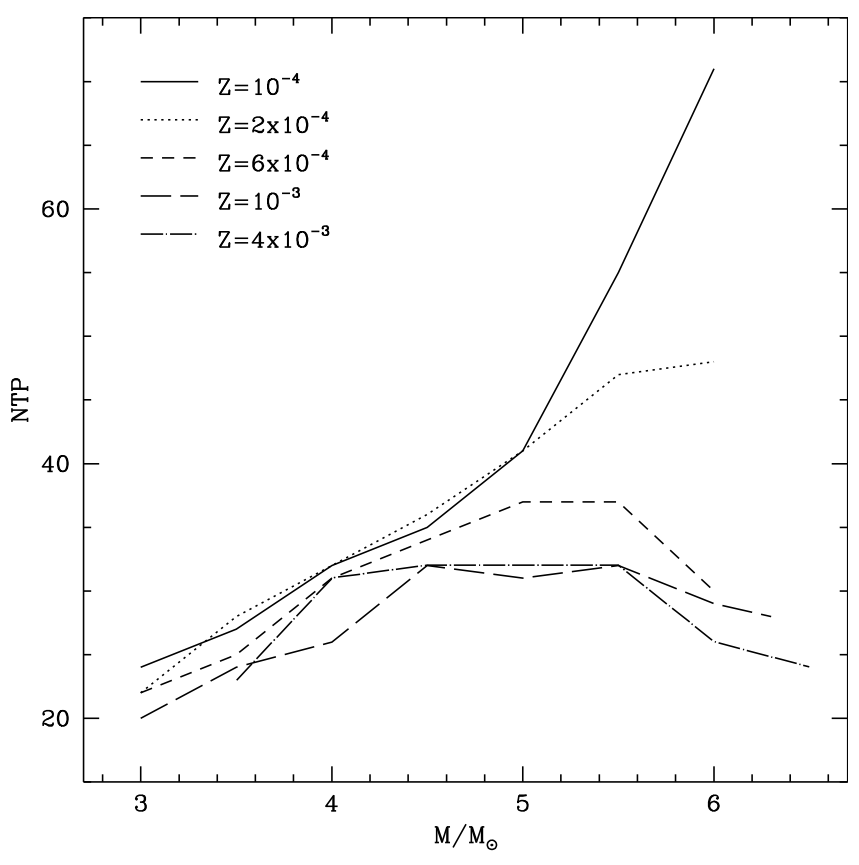

Fig. 3. Variation with initial mass in the total number of thermal pulses experienced by AGB models before the envelope is lost.

reduces the number of TPs. The main difference among models with different $Z$ is the general trend of the $M-\mathrm{NTP}$ relationship: in the lower $Z$ models, NTP increases with mass for the entire range of masses investigated, whereas in the intermediate $Z$ case NTP reaches a maximum around $4.5-5 M_{\odot}$ and declines with increasing $M$, because of the higher luminosities reached by the higher $Z$ models for the highest masses, which determines the increase in the mass-loss rate.

We therefore find an important difference between the evolutionary properties of high mass models of low and intermediate metallicity. Low $Z$ models are expected to undergo a more advanced nucleosynthesis at the bottom of their outer convective zone, and to experience more TPs.

\section{The chemical yields}

The chemistry of the ejecta of the AGBs is essential in understanding the role that these stars may play in polluting the interstellar medium; a comparison between the individual abundances of the mass ejected during their evolution and the chemical composition of the stars in GCs with the anomalous chemistry provides a good test of the reliability of the self-enrichment scenario. Columns 3 to 8 of Table 2 contain the average abundance ratios of the main elements investigated in spectroscopic surveys of $\mathrm{GCs}$, in terms of the quantity $[\mathrm{X} / \mathrm{Fe}]=\log (\mathrm{X} / \mathrm{Fe})-$ $\log (\mathrm{X} / \mathrm{Fe})_{\odot}$. The isotopic magnetic ratios of the ejecta are indicated in Cols. 11 and 12. $R(\mathrm{CNO})$ (shown in Col. 9) represents the ratio of the global $\mathrm{C}+\mathrm{N}+\mathrm{O}$ abundance of the ejecta to the initial value at the beginning of the evolution, whereas $\mathrm{C} / \mathrm{O}$ (Col. 10) indicates the ratio of the carbon to the oxygen abundance. The table also includes the results for higher $Z$ models presented in Ventura \& D'Antona (2008a,b).

\subsection{The CNO elements}

We begin our analysis by examining the abundances of the $\mathrm{CNO}$ elements, which are modified during the AGB evolution at the stellar surface by TDU and HBB.
The two panels of Fig. 4 show ${ }^{12} \mathrm{C}$ and ${ }^{14} \mathrm{~N}$ in the ejecta of our models, as a function of the initial mass $M$ of the star, for the 5 metallicities discussed. We also report the values obtained by Herwig (2004, hereinfter H04) and by Karakas \& Lattanzio (2007) to allow us to compare our own predictions with those sets of models provided by different groups; these other models have a metallicity $Z=10^{-4}$.

$[\mathrm{C} / \mathrm{Fe}]$ for low masses is high, because the increase in the carbon abundance due to the occurrence of the TDU. The trend with mass is negative, because the lower the mass of the envelope, the higher the increase in the carbon mass fraction as a consequence of the TDU. More massive models achieve HBB conditions more easily, and consequently experience depletion of the envelope ${ }^{12} \mathrm{C}$ due to proton captures at the bottom of the external convective zone; for masses close to the limit for carbon ignition, $[\mathrm{C} / \mathrm{Fe}]$ is negative (see Col. 8 of Table 1 ).

In Fig. 4, when comparing the lines corresponding to different metallicities, we see that $[\mathrm{C} / \mathrm{Fe}]$ increases as $Z$ diminishes, which is because the same quantity of carbon dredged-up to the surface corresponds to a greater increase in the carbon mass fraction in the envelope of the low $Z$ models. The number of TPs and the efficiency of the TDUs are irrelevant in determining the differences observed (see Table 1). The differences among the various metallicities vanish at the highest masses, where all sets of models tend to a limit value of $[\mathrm{C} / \mathrm{Fe}] \sim-0.7$, independently of $Z$. In these stars, TDU practically fails to operate, and strong HBB favours carbon destruction, for all Z's; the stronger depletion of carbon found at low Z's at the beginning of the AGB phase (due to the higher $T_{\text {bce }}$ 's) is counterbalanced by the higher carbon equilibrium abundances expected when the full CNO cycle is activated.

$[\mathrm{N} / \mathrm{Fe}]$ exhibits a similar trend with mass to $[\mathrm{C} / \mathrm{Fe}]$ (see the right panel of Fig. 4), because ${ }^{14} \mathrm{~N}$ is produced at the bottom of the envelope via proton capture by ${ }^{12} \mathrm{C}$ nuclei; we recall that nitrogen production requires only mild $\mathrm{HBB}$ conditions, and no activation of the full $\mathrm{CNO}$ cycle. In contrast to carbon, $[\mathrm{N} / \mathrm{Fe}]$ is positive in all cases, because it is produced and never destroyed. The nitrogen yields become $Z$-independent for the range $M \geq 5 M_{\odot}$, for the same reasons discussed in the analysis of the carbon yields.

The opposite effects of the HBB for carbon and nitrogen can be detected in the different negative slopes of the variations with mass in $[\mathrm{C} / \mathrm{Fe}]$ and $[\mathrm{N} / \mathrm{Fe}]$ : the slope of the former is much higher, whereas the decrease in $[\mathrm{N} / \mathrm{Fe}]$ with mass is more modest, since the stronger HBB acts to destroy carbon and increase $[\mathrm{N} / \mathrm{Fe}]$.

The interpretation of the average oxygen abundance ratios of the ejecta, shown in the left panel of Fig. 6, and of their trend with mass and metallicity, is less straightforward than for carbon and nitrogen. Oxygen is dredged-up in the phases following the TPs only when the TDU is very efficient. HBB leads to oxygen depletion, but, unlike carbon, this holds only when the full CNO cycle is activated at the bottom of the convective envelope, when the temperature exceeds $\sim 70-80 \mathrm{MK}$. Both factors explain why in all cases $[\mathrm{O} / \mathrm{Fe}]$ decreases with increasing mass, and becomes negative for the most massive models. For the lowest masses of our samples, for which TDU is the main cause of the change in the surface oxygen, $[\mathrm{O} / \mathrm{Fe}]$ increases as $Z$ decreases, for the same reasons outlined above. On the other hand, when $M$ increases, the higher temperatures at the bottom of the envelope attained by the low $Z$ massive models produce a stronger depletion of the surface oxygen, which is enhanced by their lower massloss rates, because, unlike their higher $Z$ counterparts, they begin to loose most of the mass of their envelope when the surface 
Table 1. Evolutionary properties of intermediate-mass models.

\begin{tabular}{|c|c|c|c|c|c|c|c|}
\hline$M / M_{\odot}$ & $\tau_{\mathrm{H}} / 10^{6}$ & $\tau_{\mathrm{He}} / 10^{6}$ & $M_{\text {core }} / M_{\odot}$ & $\log \left(L / L_{\odot}\right)_{\max }$ & $T_{\max }^{\mathrm{bce}}$ & $N_{\text {pulse }}(\mathrm{NTP})$ & $\lambda$ \\
\hline & & & $Z=10^{-4}$ & & & & \\
\hline 3.0 & 240 & 45.4 & 0.78 & 4.42 & 78 & 24 & 0.7 \\
\hline 3.5 & 174 & 29.0 & 0.81 & 4.52 & 85 & 27 & 0.7 \\
\hline 4.0 & 132 & 20.3 & 0.84 & 4.61 & 92 & 32 & 0.7 \\
\hline 4.5 & 104 & 14.7 & 0.87 & 4.69 & 98 & 35 & 0.6 \\
\hline 5.0 & 85 & 11.3 & 0.91 & 4.77 & 107 & 41 & 0.5 \\
\hline 5.5 & 70 & 9.0 & 0.96 & 4.84 & 120 & 55 & 0.5 \\
\hline \multirow[t]{2}{*}{6.0} & 60 & 7.2 & 1.01 & 4.87 & 127 & 71 & 0.3 \\
\hline & & & $Z=2 \times 10^{-4}$ & & & & \\
\hline 3.0 & 248 & 46.5 & 0.78 & 4.41 & 77 & 22 & 0.7 \\
\hline 3.5 & 179 & 29.8 & 0.81 & 4.51 & 85 & 28 & 0.7 \\
\hline 4.0 & 135 & 20.7 & 0.84 & 4.59 & 92 & 32 & 0.7 \\
\hline 4.5 & 106 & 15.1 & 0.87 & 4.68 & 98 & 36 & 0.6 \\
\hline 5.0 & 86 & 11.4 & 0.91 & 4.76 & 105 & 41 & 0.5 \\
\hline 5.5 & 72 & 9.1 & 0.95 & 4.83 & 117 & 47 & 0.5 \\
\hline 6.0 & 61 & 7.4 & 1.00 & 4.90 & 123 & 48 & 0.3 \\
\hline \multirow[t]{2}{*}{6.3} & 55 & 6.4 & 1.05 & 4.97 & 126 & 26 & 0.0 \\
\hline & & & $Z=6 \times 10^{-4}$ & & & & \\
\hline 3.0 & 265 & 50.3 & 0.77 & 4.40 & 75 & 22 & 0.7 \\
\hline 3.5 & 188 & 32.2 & 0.81 & 4.55 & 84 & 25 & 0.7 \\
\hline 4.0 & 141 & 22.5 & 0.83 & 4.59 & 91 & 31 & 0.7 \\
\hline 4.5 & 110 & 16.3 & 0.86 & 4.68 & 97 & 34 & 0.7 \\
\hline 5.0 & 89 & 12.4 & 0.90 & 4.76 & 103 & 37 & 0.6 \\
\hline 5.5 & 74 & 9.7 & 0.94 & 4.83 & 110 & 37 & 0.6 \\
\hline 6.0 & 62 & 7.9 & 1.02 & 4.94 & 116 & 30 & 0.3 \\
\hline \multirow[t]{2}{*}{6.4} & 56 & 6.6 & 1.05 & 5.00 & 118 & 29 & 0.0 \\
\hline & & & $Z=10^{-3}$ & & & & \\
\hline 3.0 & 277 & 55.0 & 0.76 & 4.39 & 73 & 20 & 0.7 \\
\hline 3.5 & 195 & 34.0 & 0.80 & 4.50 & 83 & 24 & 0.7 \\
\hline 4.0 & 146 & 23.5 & 0.83 & 4.57 & 89 & 26 & 0.7 \\
\hline 4.5 & 113 & 17.3 & 0.86 & 4.65 & 95 & 32 & 0.6 \\
\hline 5.0 & 91 & 12.8 & 0.89 & 4.72 & 101 & 31 & 0.5 \\
\hline 5.5 & 75 & 10.1 & 0.94 & 4.80 & 106 & 32 & 0.5 \\
\hline 6.0 & 63 & 8.2 & 1.00 & 4.92 & 112 & 29 & 0.3 \\
\hline \multirow[t]{2}{*}{6.3} & 58 & 7.2 & 1.03 & 4.96 & 114 & 28 & 0.3 \\
\hline & & & $Z=4 \times 10^{-3}$ & & & & \\
\hline 3.0 & 319 & 69.5 & 0.65 & 4.26 & 45 & 25 & 0.7 \\
\hline 3.5 & 220 & 42.0 & 0.77 & 4.43 & 78 & 23 & 0.7 \\
\hline 4.0 & 160 & 27.8 & 0.80 & 4.51 & 85 & 31 & 0.7 \\
\hline 4.5 & 122 & 19.6 & 0.83 & 4.60 & 89 & 32 & 0.6 \\
\hline 5.0 & 97 & 14.8 & 0.86 & 4.67 & 93 & 32 & 0.5 \\
\hline 5.5 & 79 & 11.4 & 0.89 & 4.75 & 98 & 32 & 0.5 \\
\hline 6.0 & 66 & 9.5 & 0.96 & 4.85 & 105 & 26 & 0.3 \\
\hline 6.5 & 56 & 7.8 & 1.03 & 4.95 & 110 & 24 & 0.3 \\
\hline
\end{tabular}

oxygen abundance has already diminished considerably (see the right panel of Fig. 6). The variation in $[\mathrm{O} / \mathrm{Fe}]$ with $\mathrm{Z}$ is therefore not monotonic.

In this case, at odds with findings for carbon and nitrogen, the oxygen yield varies with $Z$ even at high masses, because in low-metallicity, massive models, we expect a strong depletion of the surface oxygen, so that the overall reduction factor is almost a factor $\sim 10$ larger in the $Z=10^{-4}$ models than in the $Z=$ $10^{-3}$ case.

The sum of $\mathrm{C}+\mathrm{N}+\mathrm{O}$ abundances of the ejecta, shown in the 9th column of Table 2, diminishes with mass, as a consequence of the smaller impact of the TDU at the highest masses, and approaches unity for the most massive models; this behaviour is independent of $Z$.

The C/O ratio, reported in the 10th column of Table 2, is more sensitive to $Z$, and tends to decrease with metallicity. For small masses, HBB is negligible, and the stronger impact of the TDU in the low $Z$ models favours a higher $\mathrm{C} / \mathrm{O}$ ratio (see both panels of Fig. 4); for the masses close to the limit for carbon ignition, the strong depletion of oxygen found in the low-metallicity models (see the left panel of Fig. 6) leads to $\mathrm{C} / \mathrm{O}$ ratios sligthly below unity for $Z \geq 2 \times 10^{-4}$, and $\mathrm{C} / \mathrm{O}>1$ at $Z=10^{-4}$. The threshold metallicity is $Z \sim 2 \times 10^{-4}$, below which $\mathrm{O}$-poor yields, causing $\mathrm{C} / \mathrm{O}$ to exceed unity at the surface, are expected.

We conclude this discussion with a comparison of our yields with those by KL07 and H04. We see from Figs. 4 and 6 that our CNO abundances are systematically lower than those by KL07, so we detect a much greater CNO enhancement when the KL07 models are adopted; also, $[\mathrm{C} / \mathrm{Fe}]$ is always positive in the KL07 case. The key role in this context is played by the treatment of convection. In the models presented here, convection was modelled according to the FST treatment, whereas the KL07 models 

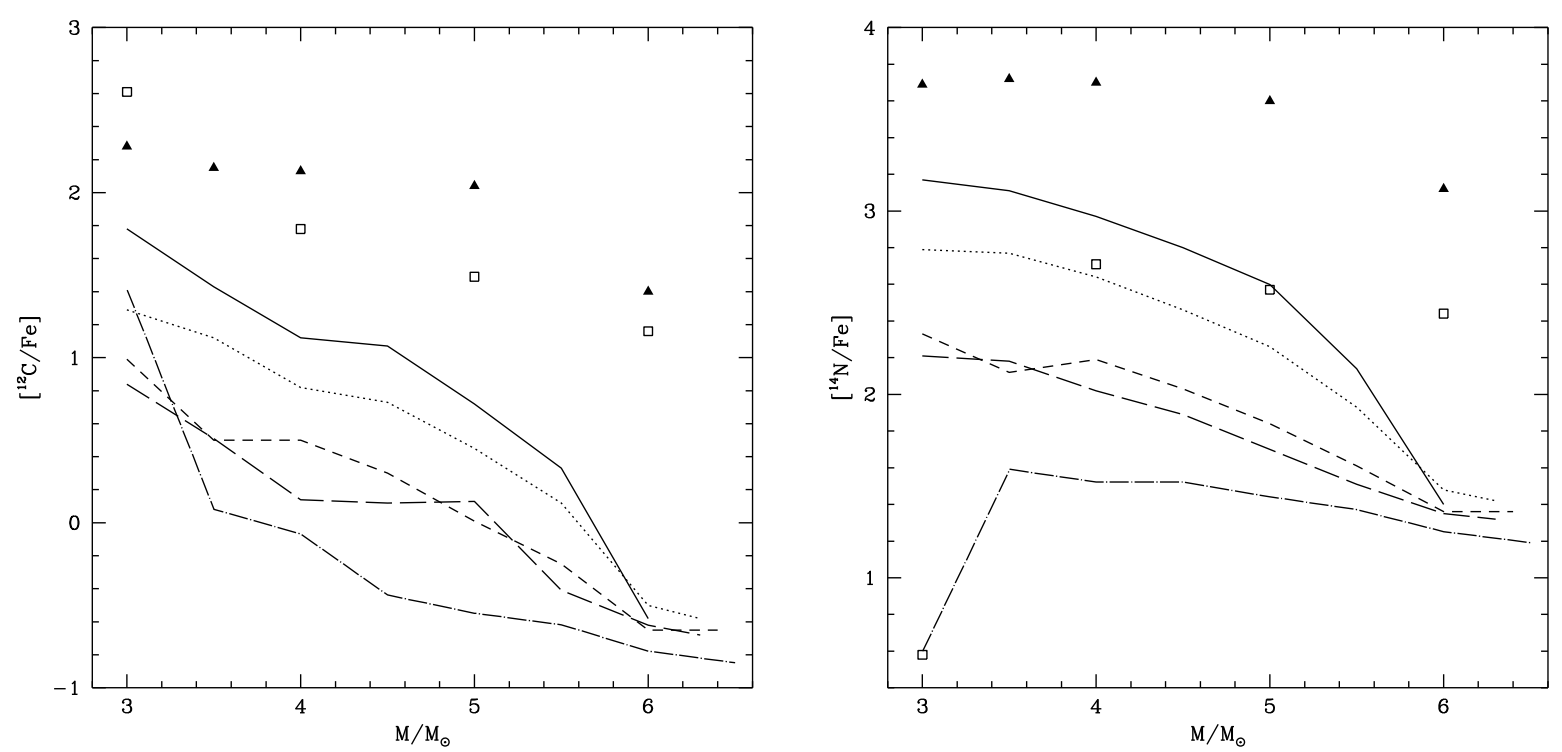

Fig. 4. Variation with the initial stellar mass in the carbon yield (left, expressed as $[\mathrm{C} / \mathrm{Fe}]$ ) and the nitrogen yield (right) of AGB models of metallicity $Z=10^{-4}$ (solid), $Z=2 \times 10^{-4}$ (dotted), $Z=6 \times 10^{-4}$ (dashed), $Z=10^{-3}$ (long dashed), and $Z=4 \times 10^{-3}$ (dotted-dashed). The full triangles indicate the yields by KL07, whereas the open squares refer to the results by Herwig (2004).

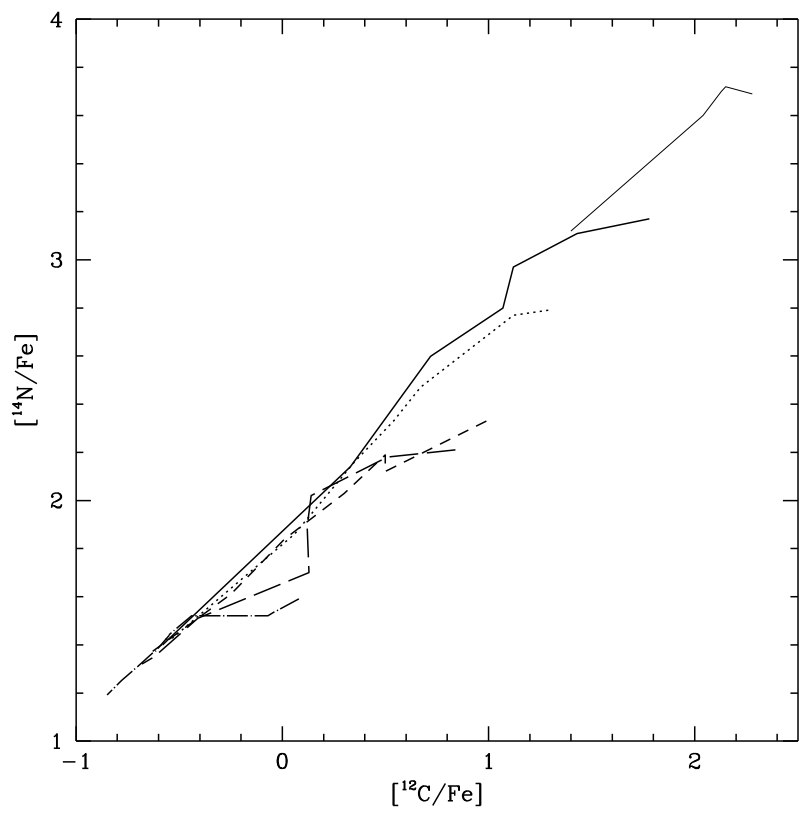

Fig. 5. The nitrogen content of the ejecta of the AGB models as a function of the carbon content. The thin solid line indicates the results by KL07.

were calculated by means of the MLT description, which provides a far less efficient description of the convective instability. As discussed in Ventura \& D'Antona (2005a), a more efficient convection model favours higher temperatures at the bottom of the convective envelope, higher luminosities, shorter life times, and a more rapid mass loss in the envelope. The above explanation probably reproduces the situation observed, since the KL07 models experience more TPs than our models. For example, our 3,4 , and $5 M_{\odot}, Z=10^{-4}$ models experience $24,32,41$ TPs respectively, compared to the 40, 76 and 138 TPs suffered by the same masses by KL07. The TDU episodes following these TPs results in large amounts of carbon being dredged-up to the surface, and the consequent increase in the abundances of all the CNO elements. The difference between our yields and those by KL07 increases with mass, because the higher temperatures attained at the base of the convective envelope lead, for a given mass, to a more efficient HBB.

The $\mathrm{C}$ and $\mathrm{N}$ yields by $\mathrm{H} 04$ are lower than those by KL07, because the $\mathrm{H} 04$ models experience a much smaller number of TPs because of the higher mass-loss rate adopted (a Blöcker 1995, law, enhanced by a factor 5 compared to ours). The amount of carbon dredged-up to the surface (which can be eventually converted to nitrogen) is thus reduced. Compared to ours, the $\mathrm{CN} \mathrm{H04} \mathrm{yields} \mathrm{are} \mathrm{higher,} \mathrm{the} \mathrm{difference} \mathrm{increasing} \mathrm{with}$ mass, because of the stronger HBB conditions experienced by our models at a given mass (for example, the average temperature at the bottom of the convective envelope for the $6 M_{\odot}$ model is $120 \mathrm{MK}$ in our case, and $100 \mathrm{MK}$ in H04). The difference in the oxygen yields (see the left panel of Fig. 6) also increases with mass between us and $\mathrm{H} 04$ models. In interpreting the differences between our results and those by $\mathrm{H} 04$ we emphasize that the different efficiencies of the TDU also plays a role: in our models, no extra-mixing is assumed from the bottom of the convective envelope, whereas an exponential overshooting is adopted in the H04 models.

The differences due to the metallicity and to the treatment of convection can be more easily understood when the yields of the models are shown on the $\mathrm{C}-\mathrm{N}$ plane; this will also be of help when discussing the self-enrichment scenario on the basis of the observed C-N abundances of SGB stars in M 15. The [N/Fe] values of the 5 sets of models discussed here and the KL07 models are shown as a function of $[\mathrm{C} / \mathrm{Fe}]$ in Fig. 5. We note that all models correspond approximately to a straight line in the $\mathrm{C}-\mathrm{N}$ plane, confirming that $\mathrm{CN}$ cycling operates at the bottom of the envelope. The KL04 models are located in the right-upper portion of the plane, whereas our models occupy the lower region. In agreement with the discussion after our presentation of the carbon and nitrogen yields, we find that, independently of $Z$, all curves corresponding to the different metallicities converge to the same locus on the plane, which is $([\mathrm{C} / \mathrm{Fe}],[\mathrm{N} / \mathrm{Fe}]) \sim(-0.7,1.3)$. This 
Table 2. Chemical yields of intermediate-mass models.

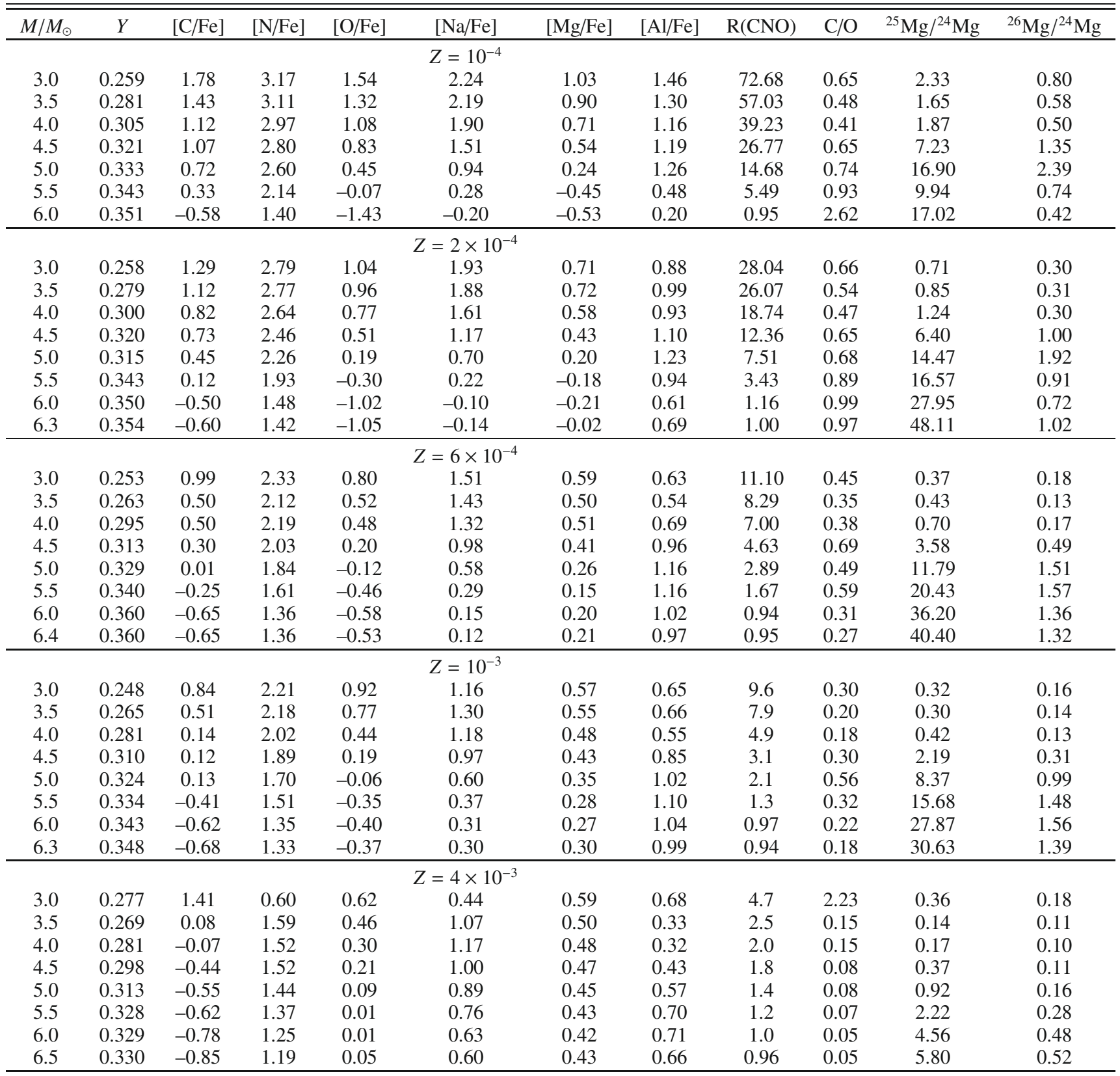

is untrue for the KL07 models, for which a minimum increase in carbon and nitrogen of a factor of $\sim 20$ and $\sim 1000$ respectively are found.

\subsection{Sodium}

The sodium content of the ejecta of AGBs is important in understanding the differences between the GCs stars in the selfenrichment scenario: the oxygen-sodium anticorrelation is by far the most well studied anticorrelation that was confirmed by a deep spectroscopic analysis of many GCs (Carretta et al. 2006).

The surface abundance of sodium is determined by two processes that change the surface chemistry of AGBs. By the dredge-up of ${ }^{22} \mathrm{Ne}$ from the ashes of the $3 \alpha$ burning shell TDU tends to increase the surface sodium mass fraction, and HBB favours a further increase in the surface sodium as far as the temperature at the bottom of the envelope does not exceed $\sim 70 \mathrm{MK}$, above which the destruction channel dominates. These considerations allow us to understand the tracks shown in Fig. 7, which show the sodium content (in terms of $[\mathrm{Na} / \mathrm{Fe}]$ ) as a function of the initial mass of the ejecta in the various sets of models presented here; we also show the results by KL07 and H04.

The negative trend of $[\mathrm{Na} / \mathrm{Fe}]$ with $M$ found for all $Z$ 's is caused, as it was for the carbon and oxygen, by our observing in the lowest mass models of the effects of TDU mainly, which acts to enhance the surface sodium, whereas for high masses, HBB is the dominant mechanism, producing lower sodium contents the higher is the temperature at the bottom of the convective envelope. As for oxygen, we do not find a monotomic behaviour with $Z$, because at low metallicities, the bottom of the surface convective zone of massive AGBs becomes hot, and favours sodium depletion. 

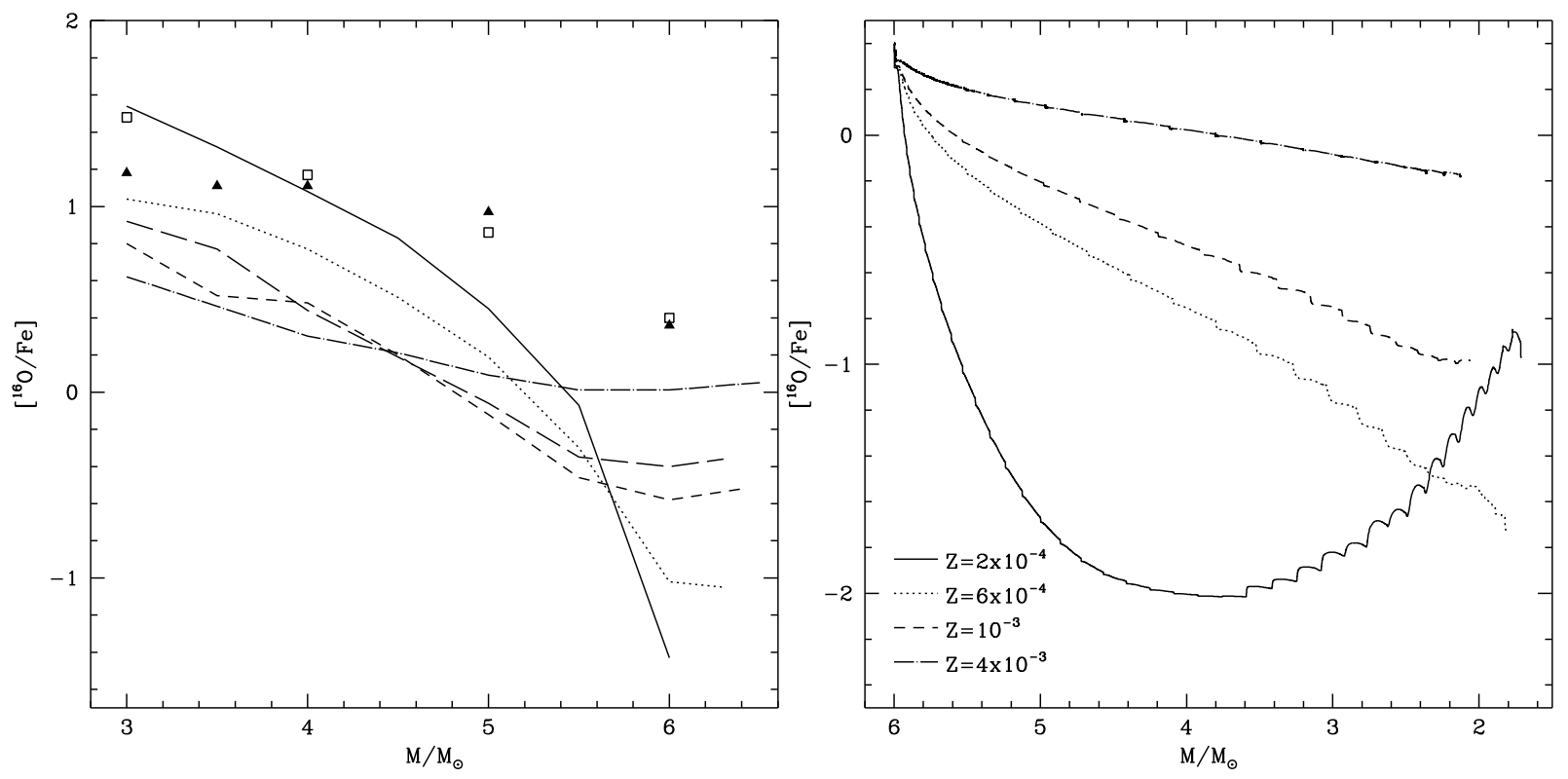

Fig. 6. Left: the oxygen content of the ejecta of the models discussed in the paper as a function of the initial mass. The individual metallicities and the results by H04 and KL07 are indicated by different labels, whose meaning is the same as Fig. 4. Right: the variation of the surface oxygen abundance during the evolution of massive AGB models of mass $6 M_{\odot}$ for various metallicities.

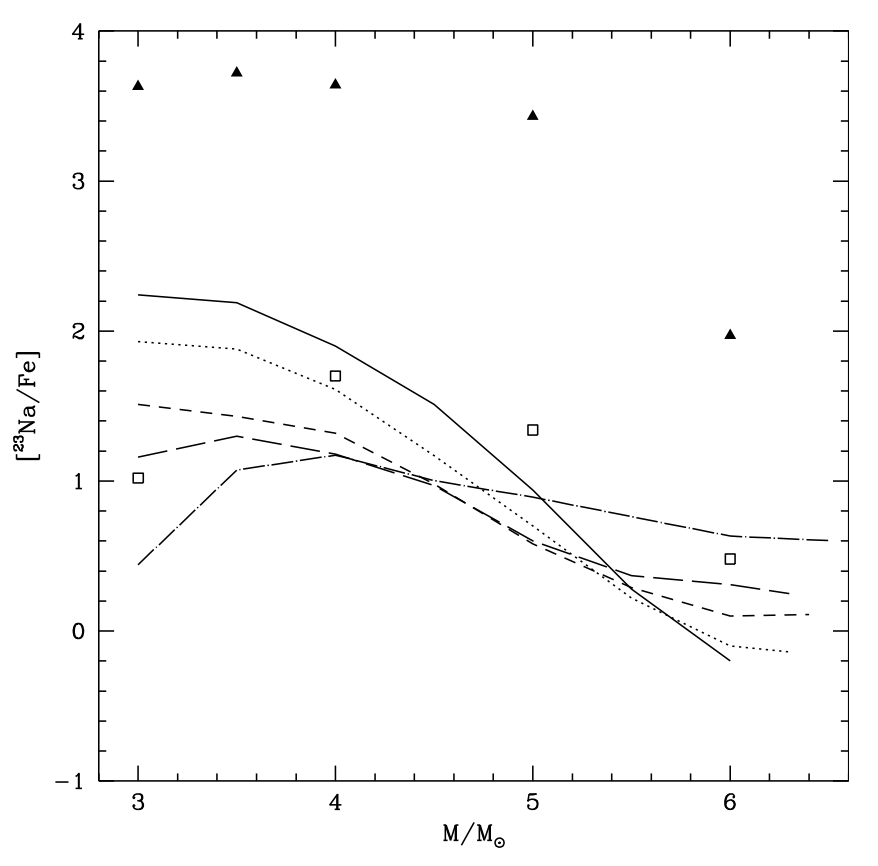

Fig. 7. The sodium content of the ejecta of the AGB models as a function of the carbon content. The meaning of the different labels is the same as in Fig. 4.

By comparing our results with the yields by KL07, we confirm that it is the temperature in the innermost layers of the outer convective zone which drives physically and chemically the evolution of AGBs. The KL07 sodium yields are systematically higher than ours, because of their more numerous TDU episodes, which carries more ${ }^{22} \mathrm{Ne}$ to the surface, that is then available for conversion to sodium during the quiescent $\mathrm{CNO}$ burning phase. The milder efficiency of the HBB prevents then the strong depletion of the surface sodium in the more massive models, that we found in our computations.

Our sodium yields are more similar to those by H04, whereas the slope of the $[\mathrm{Na} / \mathrm{Fe}]$ versus mass relation is steeper in our case, because of the strong sodium depletion achieved in our high mass models. Unlike the CNO elements, we note that the reliability of the results obtained for sodium is extremely low, because the cross-sections of the three key-reactions relevant to the determination the sodium yield, i.e., the creation channel of ${ }^{22} \mathrm{Ne}(\mathrm{p}, \gamma){ }^{23} \mathrm{Na}$, and the two destruction channels ${ }^{23} \mathrm{Na}(\mathrm{p}$, $\gamma)^{24} \mathrm{Mg}$ and ${ }^{23} \mathrm{Na}(\mathrm{p}, \alpha){ }^{20} \mathrm{Ne}$, are uncertain by up to 3 orders of magnitude (Hale et al. 2002, 2004). According to the crosssections adopted, Ventura \& D'Antona (2008a) showed that the average sodium mass fraction of the yields in their most-massive intermediate-metallicity AGB models should increase or diminish relative to the initial abundance (see their Fig. 9). A more detailed investigation of this topic was completed by Izzard et al. (2007), who showed that the sodium yields of AGB models of low metallicity was highly uncertain, and that the poor knowledge of the cross-sections of the relevant proton capture reactions determine the uncertainty associated with the expected sodium yield of the order of $\sim 10-100$.

\subsection{Aluminium}

Aluminium appears to correlate with sodium and be anticorrelated with oxygen and magnesium in stars belonging to GCs, where clear star-to-star differences are observed. Among all the light elements involved in the commonly studied abundance patterns, aluminium is the species showing the largest spread, the maximum detected abundances being of the order of $[\mathrm{Al} / \mathrm{Fe}] \sim 1$ in all the stars showing a significant depletion of oxygen and an enhancement of sodium. In an analysis of the surface chemistry of TO and SGB stars in the GC NGC 6752, Gratton et al. (2001) found stars with $[\mathrm{Al} / \mathrm{Fe}]=1$. A similar aluminium enhancement was also detected by Sneden et al. (2004) in giants of low and high gravity in the two GCs M 3 and M 13. A study by Smith et al. (2005) of M 4, which was unfortunately limited to giants, demonstrated the presence of aluminium-rich stars of $[\mathrm{Al} / \mathrm{Fe}]=0.8$. Finally, Sneden et al. (1997) detected a few stars in the GCs M 15 and M 92 (both clusters having a metallicity that 
is more appropriate for this investigation) with $[\mathrm{Al} / \mathrm{Fe}] \sim 1$, although even in this case, the study was focused on bright giants.

Aluminium is produced in AGBs by HBB and the activation of the $\mathrm{Mg}-\mathrm{Al}$ chain, which requires temperatures approximately of $80 \mathrm{MK}$. TDU also produces an indirect aluminium enhancement, because the two magnesium isotopes produced in the $3 \alpha$ burning shell are convected to the surface, where they synthesize aluminium via proton capture. Ventura \& D'Antona (2008a) found that, when the upper limits to the cross-sections of the proton capture reactions by the heavy magnesium isotopes are adopted, the most massive among their AGB models of intermediate metallicity produce significant amounts of aluminium, with an average increase of a factor $\sim 10$, in agreement with observations of GCs of that chemistry, such as M 3 and M 13 (see the corresponding lines in Table 2). The results by Ventura \& D'Antona (2008b, obtained by using the same upper limits for the above mentioned proton-capture reactions, as also in the present investigation) confirmed the possibility of producing aluminium at the surface of massive AGBs also for more metal-rich models, though in this case the maximum enhancement found was $[\mathrm{Al} / \mathrm{Fe}] \sim 0.7$.

The present results confirm the outcome of the aforementioned investigations, as can be seen by noting the results concerning the magnesium and aluminium yields in Table 2. Here, we also show the ${ }^{25} \mathrm{Mg} /{ }^{24} \mathrm{Mg}$ and ${ }^{26} \mathrm{Mg} /{ }^{24} \mathrm{Mg}$ ratios, which are extremely dependent on the assumed rates of the proton-capture reactions by the two heavy magnesium isotopes. In Table 2 we note that for low-metallicity models with $Z<6 \times 10^{-4}$, the trend [Al/Fe] versus $M$ is not monotonic for the complete range of masses investigated. For $Z=2 \times 10^{-4}$ we find an aluminium enhancement by a factor of $\sim 10$ for masses $M \leq 5 M_{\odot}$, whereas slightly lower values $([\mathrm{Al} / \mathrm{Fe}] \sim 0.6-0.8)$ are predicted for masses close to the limit for carbon ignition. This effect is due to the very high temperatures reached by these models at the bottom of their external convective zone, which becomes sufficient to activate efficiently the proton-capture reaction by ${ }^{27} \mathrm{Al}$ nuclei. On the basis of these computations, we infer that the maximum enhancement reached by the most massive models of low metallicity is of the order of $[\mathrm{Al} / \mathrm{Fe}] \sim 0.7$.

\section{The observed abundance patterns in low metallicity globular cluster stars}

Ventura \& D'Antona (2008a,b) compared the yields of AGB models with the abundance patterns observed by deep spectroscopic investigations of $\mathrm{GC}$ stars of $[\mathrm{Fe} / \mathrm{H}] \sim-1.3$ and $[\mathrm{Fe} / \mathrm{H}] \sim-0.7$. We extend the analysis here to lower $Z$, by using the new models presented in the previous sections.

Unfortunately, data for the lowest metallicity clusters are scarce, and few data are available for non-evolved or almost nonevolved stars.

We use data for the clusters M 15 and NGC 6397. Harris (2003) lists $[\mathrm{Fe} / \mathrm{H}]=-2.26$ for $\mathrm{M} 15$, and $[\mathrm{Fe} / \mathrm{H}]=-2.0$ for NGC 6397. For the latter cluster, we also have a measure of the $\alpha$-enhancement, $[\alpha / \mathrm{Fe}]=+0.34 \pm 0.02$ (Gratton et al. 2003), while Strömgren photometry provides $[\mathrm{Fe} / \mathrm{H}]=-1.83 \pm 0.04$ and $[\alpha / \mathrm{Fe}]=0.3$ (Anthony-Twarog $\&$ Twarog 2000). In our $\alpha$-enhanced models, $Z=2 \times 10^{-4}$ corresponds to $[\mathrm{Fe} / \mathrm{H}]=-2.3$, and $Z=6 \times 10^{-4}$ corresponds to $[\mathrm{Fe} / \mathrm{H}]=-1.82$, so we use these two sets for our respective comparisons.

Sneden et al. (1991) detected a clear spread in the surface oxygen abundances of giant stars in the two metal-poor GCs M 15 and M 92. Their Fig. 12 shows that there may be some correlation between the oxygen abundance and the evolutionary status of the individual objects. More luminous stars, closer to the RGB tip, show the lowest oxygen abundances, although a wide spread is found at all luminosities. For the few stars for which the nitrogen abundance was also measured, a N-O anticorrelation exists, which is indicative of material processed by proton-capture nucleosynthesis. A more complete analysis of M 15 giants, focusing on the abundances of oxygen, sodium, magnesium, and aluminium, was presented by Sneden et al. (1997), the main result of which was that oxygen is anticorrelated with sodium, and magnesium anticorrelates with aluminium, which again are signatures of proton-capture processing. Carretta et al. (2005) examined the CNO abundances of several clusters, including the low $Z$ cluster NGC 6397. Data for dwarf and subgiants are available for CNO and Na. Finally, Cohen et al. (2005) analyzed a large amount of spectra of subgiant stars at the base of the RGB of M 15, focusing on the abundances of carbon and nitrogen.

A direct comparison between the our model yields and observations can test the self-enrichment scenario only if the observed abundances were not altered by any in situ process to ensure that the chemistry observed is identical to when the stars formed. This is surely the case for the Cohen et al. (2005) and Carretta et al. (2005) data, because the sample of stars observed are in evolutionary stages for which advanced nucleosynthesis in their internal regions can be excluded because of their low internal temperatures. The same conclusion does not necessarily hold for the two surveys by Sneden and coworkers, involving only bright giants close to the RGB tip. Canonical stellar models predict that the first dredge-up is the only episode prior to the helium flash that can alter their surface chemistry, changing only the abundances of the two carbon isotopes and nitrogen, and leaving unchanged the abundances of heavier nuclei. However, investigations by Sweigart \& Mengel (1979) and Cavallo et al. (1998) showed that in low $Z\left(\leq 5 \times 10^{-4}\right)$ giants rotationallydriven meridional circulation-currents, if present, can penetrate deeply into internal regions, because the entropy barrier associated with the drop in the hydrogen content, preventing the inward penetration of the surface convetive layer, is more internal. Based on 3D numerical simulations, Eggleton et al. (2008) found that a deep mixing mechanism associated with a small molecular-weight inversion must operate in all low-mass giants. An observational indication that luminous giants have lower oxygen abundances came from the early M 13 data by Sneden et al. (2004). Furthermore, Carretta et al. (2006) showed that the lowest oxygen abundances in NGC 2808 are those only of giants. Based on these indications, D'Antona \& Ventura (2007) reproduced the most extreme oxygen and sodium abundances by applying deep extra-mixing from the bottom of the surface convective envelope of giant stars, based on the assumption that stars born from the ashes of an early generation of massive AGBs should have a higher helium mass-fraction: this would then decrease the height of the aforementioned entropy barrier preventing the inward penetration of the convective envelope. These results indicate that the surface chemistry of low- $Z$ giants can be altered during RGB evolution, particularly after the CNO burning shell crosses the chemical discontinuity remaining after the first dredge-up episode. Based on this, we proceed to a full, detailed comparison between our theoretical yields and the observed abundance patterns mainly for the case of the $\mathrm{O}-$ $\mathrm{Na}$ anticorrelation in NGC 6397 and in the case of the M $15 \mathrm{C}$ and $\mathrm{N}$ data by Cohen et al. (2005). In spite of the warnings issued above, we also comment on the $\mathrm{O}, \mathrm{Na}, \mathrm{Mg}$, and $\mathrm{Al}$ data by Sneden et al. (1997) for M 15 giants. 


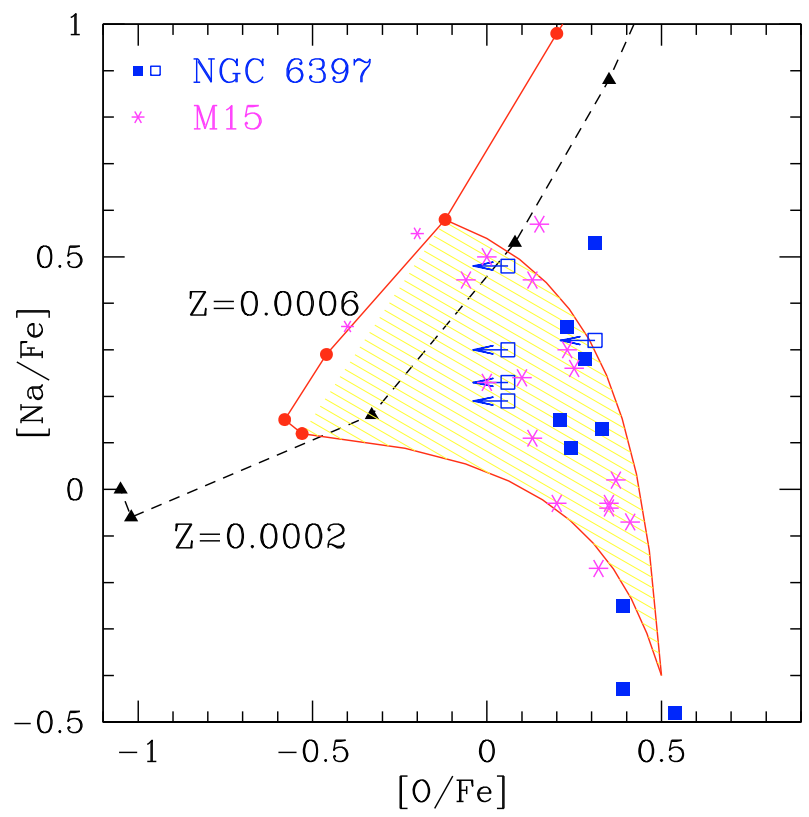

Fig. 8. We show the $\mathrm{Na}-\mathrm{O}$ data for TO and $\mathrm{SGB}$ stars belonging to the GC NGC 6397, according to Carretta et al. (2005). Data for M 15 giants by Sneden et al. (1997) are also plotted as asterisks. The triangles and dashed line are the yields of the $Z=0.0002$ models, while the dots and full line represent the yields of the $Z=0.0006$ models $(6.4,6.0,5.5$, 5.0 and $4.5 M_{\odot}$ models are plotted in both cases). The latter metallicity is the most adequate for NGC 6397, considering the $\alpha$-enhancement. In order to reproduce the data points, it is necessary to consider dilution curves between the 6.4 and $5 M_{\odot}$ yields and an initial composition represented by the vertex of the cone, and correspondig to the first generation composition.

\subsection{The O-Na anticorrelation in NGC 6397}

Figure 8 shows the Na versus O data for NGC 6397 subgiants and dwarf stars listed by Carretta et al. (2005). Upper limits are indicated by open squares with arrows. We also report the data of M 15 giants by Sneden et al. (1997). An examination of their Fig. 4 shows that the data for M 92 occupy the same region of the $\mathrm{Na}-\mathrm{O}$ plane. The $\mathrm{Na}-\mathrm{O}$ yields of our models from Table 2 are reported in the figure. It is evident that the models predict much lower oxygen abundance, when the SG stars are formed from undiluted matter from the AGB stars. Nevertheless, we can reproduce the abundances of these clusters if we hypothesize that the matter from the ejecta of stars of initial mass 6.4 to $5 M_{\odot}$ is diluted with pristine matter at the level of $\sim 50 \%{ }^{2}$. The two solid curves in the figure represent the composition of matter of initial abundance as the 6.4 and $5.0 M_{\odot}$ ejecta, diluted with different amounts of pristine matter. If we assume that dilution occurs, the helium content of the SG stars can be inferred by considering the helium abundance given in Table 2 for the 6.4 and $5 M_{\odot}$ stars $(Y=0.33-0.36)$ and diluting this with half the matter having the Big Bang initial abundance of $Y=0.24$. The resulting $Y \sim 0.28-0.30$ is consistent with the "short" blue HB of this cluster: an extremely blue HB would be obtained if the AGB matter forming the SG had been undiluted. If the helium content of the SG were as high as $Y=0.36$, both a very blue

2 The dynamical models by D'Ercole et al. (2008) showed that the pristine gas remaining in the outskirts of the globular cluster at the end of the supernova epoch, falls back into the cluster core, where it mixes with the AGB ejecta and forms the SG stars. Depending on the cluster mass and history, the SG can consist totally or in part of AGB ejecta, or its formation may start directly in the mixed gas.

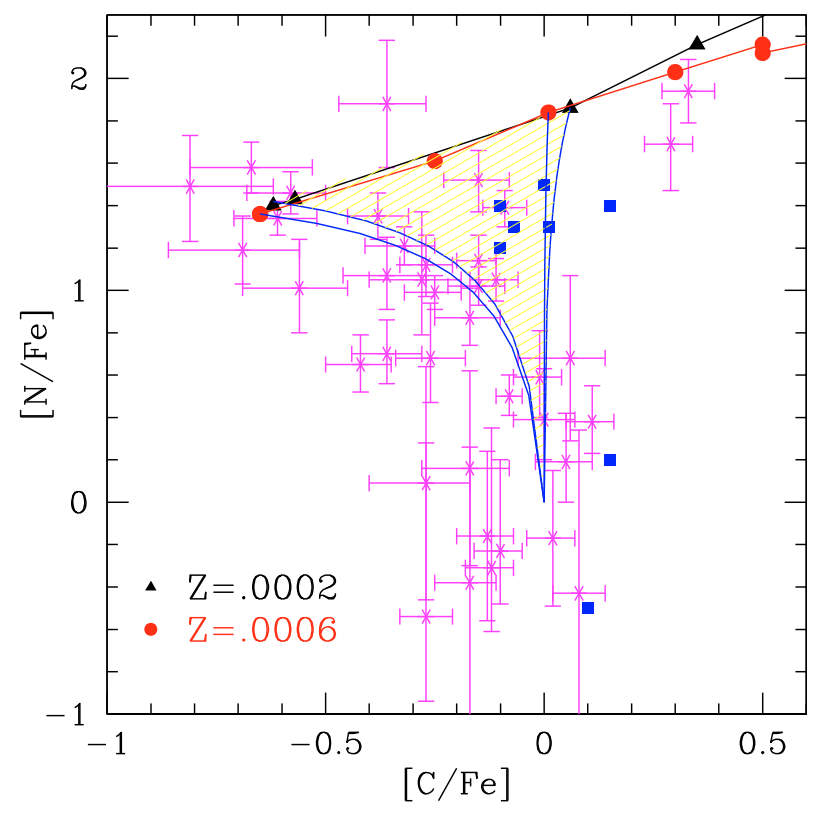

Fig. 9. Data points for TO and SGB stars belonging to M 15 (Cohen et al. 2005) are shown as asterisks with error bars. Data for NGC 6397 from Carretta et al. (2005) are shown as full squares. The lines with triangles $\left(Z=2 \times 10^{-4}\right)$ and $\operatorname{dots}\left(Z=6 \times 10^{-4}\right)$ are the theoretical abundances, for masses $\leq 6.4 M_{\odot}$. The cones corresponds to the regions allowed by dilution of the ejecta from masses $6.4 \leq M / M_{\odot} \leq 5.5$ with pristine matter having the composition of the cones vertex.

HB (e.g. D'Antona \& Caloi 2004) and a division of the main sequence (Piotto et al. 2007), as in the cluster NGC 2808, would appear in the data.

The few M 15 data shown in Fig. 8 refer to giants, and our models for the metallicity $Z=2 \times 10^{-4}$ that are appropriate for M 15, predict SG stars of low sodium and very low oxygen abundances, depending on reaction rates. There are presently insufficient available data to test these predictions. As for sodium, we have already noticed that the initial value of ${ }^{20} \mathrm{Ne}$ in the models and the cross-sections can affect by a significant amount the final abundance. In contrast, the oxygen yield cannot change, unless we consider the efficiency of convection again in our models. In the next section, we discuss the $\mathrm{C}$ and $\mathrm{N}$ abundances in $\mathrm{M} 15$, and see that some of its subgiants have abundances consistent with our most massive models, so that we should expect to find very low oxygen abundances in these same stars, although data are not available to falsify the model.

\subsection{The $C-N$ anticorrelation in $M 15$}

The main finding of Cohen et al. (2005), shown in Fig. 9 and based on the abundances included in their Table 2, is the existence of a clear anticorrelation between the surface abundances of carbon and nitrogen. A group of stars (in the left-upper part of Fig. 9) exhibit a large enhancement of nitrogen ([N/Fe] 1.4) and a strong depletion of ${ }^{12} \mathrm{C}(([\mathrm{C} / \mathrm{Fe}] \geq-0.8)$. In Fig. 9, we also include Carretta et al. (2005) data for the cluster NGC 6397. Superimposed on the observed points, we show our dilution region, obtained by assuming different degrees of dilution between matter of the chemical compositions of models with $Z=2 \times 10^{-4}$ and $Z=6 \times 10^{-4}$, and masses between 6.4 and $5.5 M_{\odot}$, and gas 
with the original "standard" chemistry ${ }^{3}$. We see that the theoretical carbon and nitrogen abundances reproduce satisfactorily the observed patterns.

The small amount of available data for NGC 6397 are consistent with the dilution expected from the oxygen and sodium data shown in Fig. 8. In contrast, a direct comparison between Figs. 8 and 9 cannot be made for M 15, as the oxygen and sodium abundances refer to different stars. Nevertheless, the $\mathrm{C}$ and $\mathrm{N}$ data require that the stars with the lowest carbon abundances consist of undiluted ejecta of the most massive AGBs. We predict that these stars should have very low oxygen abundances.

In comparing their observed abundances with the previous models by Ventura et al. (2002, in good agreement with the present yields), Cohen et al. (2005) argued that the agreement is only qualitative, since the models fail to reproduce the significant nitrogen enhancement inferred from the difference between their lowest and highest values $([\mathrm{N} / \mathrm{Fe}] \sim-0.5$ and $[\mathrm{N} / \mathrm{Fe}] \sim 1.5$ in their Fig. 4). This conclusion is, however, misleading. Nitrogen, regardless of the exact mixture used, is the least abundant of the $\mathrm{CNO}$ elements, so that the final nitrogen yield (proportional to the overall $\mathrm{C}+\mathrm{N}+\mathrm{O}$ abundance, under strong HBB conditions) is almost independent of the initial nitrogen adopted, provided that the same carbon and (expecially) oxygen abundances are used. We confirmed this conclusion by calculating a $6 M_{\odot}$ model of metallicity $Z=2 \times 10^{-4}$ with an initial abundance of nitrogen $[\mathrm{N} / \mathrm{Fe}]=-0.5$. We find that the $\mathrm{N}$ yield as a function of mass is practically the same (0.05 dex lower) as in Table 2.

Interestingly, Cohen et al. (2005) compare their C-N trend for M 15 with those from other clusters of higher metallicity, and find that, independently of $[\mathrm{Fe} / \mathrm{H}]$, the observations encompass the same range of values of $[\mathrm{C} / \mathrm{Fe}]$ and $[\mathrm{N} / \mathrm{Fe}]$. For models including strong $\mathrm{HBB}$ with full $\mathrm{CNO}$ burning in which the effects of TDU are negligible, this is consistent with our previous finding that the yields of carbon and nitrogen become $Z$-independent, converging to the most extreme values detected by spectroscopic studies quoted by the authors.

\subsection{The $M g-A l$ anticorrelation}

Few data are available, but Sneden et al. (1997) measured Mg and $\mathrm{Al}$ abundances for the same sample of M 15 giants for which we have shown the $\mathrm{O}-\mathrm{Na}$ data in Fig. 8. Figure 3 in their paper replicates the trend of $\mathrm{Na}, \mathrm{Mg}$, and $\mathrm{Al}$ with respect to $[\mathrm{O} / \mathrm{Fe}]$ in this sample. Our data in Table 2 for $Z=2 \times 10^{-4}$ are consistent with these trends: low oxygen corresponds to low magnesium and high sodium. In this case, however, we must also not consider the most extreme yields for the $6.4 M_{\odot}$ and $6.0 M_{\odot}$, since no stars of very low oxygen abundance (and low sodium abundance, although this result is less certain) have yet been found in the cluster. The lack of low oxygen abundance stars in M 15 requires explanation, although we should search for stars of very low oxygen in very low metallicity clusters. If none is found, the present models either achieve too strong HBB, or the problem depends on the modalities of formation of the lowest metallicity clusters, where some other mechanism prevents the direct formation of SG stars from the ejecta of the most massive AGBs.

\footnotetext{
3 The mass range from which we think the polluting matter comes from is quite small. This is indeed the reason why models for the formation of multiple populations in globular clusters require that the firstgeneration initial mass must be much higher than the mass observed today (e.g., D'Ercole et al. 2008).
}

\subsection{The $\mathrm{C} / \mathrm{O}$ ratio in low metallicity environments}

Table 2 shows that the $\mathrm{C} / \mathrm{O}$ ratio in the ejecta of the lowest metallicity AGB models becomes increasingly higher as the mass of the model increases, and is definitely higher than in the $6 M_{\odot}$ model of $Z=10^{-4}$. During their life, the most massive AGBs of low metallicity therefore become carbon stars, although this is not because of the dredge-up of carbon, but to the very strong oxygen depletion caused by proton captures on oxygen nuclei during the hot bottom burning. The carbon-star stages of evolution in massive low- $Z$, AGBs was already discussed in Ventura et al. (2002) $)^{4}$ A full explanation of the O-Na anticorrelation would require the AGB matter to be diluted with pristine cluster matter in most of the SG stars (Ventura \& D'Antona 2008), so it is unclear how high the $\mathrm{C} / \mathrm{O}$ ratios are in the low- $Z$ clusters. A full discussion of these results is postponed to an analysis including results of modeling at lower metallicities. Here we restrict ourselves to a few speculative issues.

- Table 2 shows that the ratio C/O from the massive AGB ejecta is higher the lower is the metallicity of the cluster; these ejecta are also expected to be extremelly oxygen-poor. This theoretical prediction requires observational verification.

- It is curious that clusters of metallicity $<2 \times 10^{-4}$, in which we should expect some second-generation stars have $\mathrm{C} / \mathrm{O}>1$, do not exist. There are, however, many carbon-rich halo stars with metallicities lower than $10^{-4}$ (e.g., Beers \& Sommer-Larsen 1995; Beers \& Christlieb 2005). Do carbonrich grains form from the carbon that is not locked in $\mathrm{CO}$, even if the metallicity is so low? Does this affect the formation of second-generation stars? Dust formation in the envelopes of giants is found in C-rich models of stellar envelopes for metallicities at least as low as that of the Small Magellanic Cloud (Wachter et al. 2008).

- In the ejecta with $\mathrm{C} / \mathrm{O}>1$, the carbon abundance is low $([\mathrm{C} / \mathrm{Fe}]<0$, Table 2$)$, so that observational counterparts cannot be identified among CEMP (carbon-enhanced metalpoor) stars, defined as stars for which $[\mathrm{C} / \mathrm{Fe}]>1$ (Beers $\&$ Christlieb 2005). These models also have extremely low $\mathrm{C} / \mathrm{N}$ ratios, since the nitrogen abundance is very large due to HBB (both carbon and oxygen contributing to its value). These are then the NEMPs (nitrogen-enhanced metal-poor stars) according to the definition by Johnson et al. (2007), who failed to find any of these stars by searching among extremely metal poor stars. We note that a ratio $[\mathrm{C} / \mathrm{N}] \simeq-2$ is expected from Table 2, although not even ratios of less extreme value such as $[\mathrm{C} / \mathrm{N}] \simeq-1$ expected from the models by Herwig (2004) were found in this survey. The presence of enhanced $\mathrm{C}$ and $\mathrm{N}$ in extremely metal-poor lowmass stars presently observed is generally attributed to mass transfer from a previously evolving AGB companion (e.g., Lucatello et al. 2005). Johnson et al. (2007) attributed the lack of NEMPs to the shortage of binaries with the extreme mass ratios required to produce them.

- The presence of high $\mathrm{C} / \mathrm{O}$ ratios - and of low total $\mathrm{C}$ and $\mathrm{O}$ abundances, could be inferred from the properties of gas and dust in the circumnuclear region of the very high redshift QSO (e.g., Maiolino et al. 2004).

\footnotetext{
${ }^{4}$ A different mechanism producing a C-rich stage in massive AGBs was described by Frost et al. (1998). In that case, the occurrence is due to the combined action of the third dredge-up and of mass loss that reduces the action of HBB in the latest phases of the AGB life, and observational counterparts may have been identified in the obscured, C-rich stars of high bolometric luminosity (van Loon et al. 1999).
} 


\section{Conclusions}

We have presented new evolutionary models focused on the AGB phase of intermediate-mass stars $\left(M \geq 3 M_{\odot}\right)$ with metallicites $10^{-4} \leq Z \leq 6 \times 10^{-4}$. These results complete previous investigations of higher $Z$ AGB models by our group. We found that low $Z$ models, due to the lower amount of CNO present in their H-burning layers, attain higher temperatures both in the CNO burning shell and at the bottom of their surface convective region, and reach HBB conditions more easily than their higher $Z$ counterparts. For a given luminosity, they suffer a lower mass loss (due to their smaller radii), and the total number of thermal pulses that they experience is thus higher. In the low-mass regime $\left(M<5 M_{\odot}\right)$, where the effects of the third dredge-up dominate over those of hot bottom burning, the sum of $\mathrm{C}+\mathrm{N}+\mathrm{O}$ along with $\mathrm{Na}, \mathrm{Mg}$, and $\mathrm{Al}$ increase with decreasing $Z$ (the $\mathrm{Al}$ production in these models is favoured by the use of the upper limits to the cross-sections of the proton-capture reactions by the magnesium isotopes). For masses $M>5 M_{\odot}$, HBB takes over as the main physical process changing the surface chemistry, and the abundances of those elements that at high temperatures suffer destruction by proton capture, primarily oxygen and sodium, diminish for decreasing $Z$; conversely, the content of carbon and nitrogen are seen to be $Z$-independent for masses close to the limit for carbon ignition in degenerate conditions. A comparison of our yields with those by a different research group confirms that the treatment of convection plays the most striking role in determining the essential evolutionary properties of these class of objects.

The O-Na anticorrelation shown by NGC 6397 stars can be explained if dilution at a level of $\sim 50 \%$ between the mass ejected by AGBs and pristine matter is adopted; this is also in good agreement with the resulting helium mass fraction of the SG stars, $Y \sim 0.28-0.30$, which is consistent with the morphology of the HB for this cluster.

A comparison with spectroscopic results of $\mathrm{CN}$ abundances in low-luminosity M 15 giants shows that the chemistry of the theoretical ejecta of our models agree with the most extreme chemistries observed, i.e., with those of the stars showing the highest nitrogen enhancement and carbon reduction. Even at these low metallicities, this seems to confirm that a selfenrichment by massive AGBs is responsible for the star-to-star differences observed, although more observational results are needed before drawing more robust conclusions.

Very oxygen-poor stars $([\mathrm{O} / \mathrm{Fe}] \sim-1)$ are predicted to exist as SG stars formed by the winds of our most massive models of $Z=2 \times 10^{-4}$, at least for the most massive very low-metallicity GCs.

Acknowledgements. The authors are grateful to the anonymous referee for his detailed and careful reading of this work, that greatly helped improving the quality of the manuscript.

\section{References}

Angulo, C., Arnould, M., Rayet, M., et al. 1999, Nucl. Phys. A, 656, 3 Bedin, L. R., Piotto, G., Anderson, J., et al. 2004, ApJ, 605, L125 Beers, T. C., \& Christlieb, N. 2005, ARA\&A, 43, 531

Beers, T. C., \& Sommer-Larsen, J. 1995, ApJS, 96, 175 Blöcker, T. 1995, A\&A, 297, 727

Blöcker, T., \& Schönberner, D. 1991, A\&A, 244, L43

Busso, G., Cassisi, S., Piotto, G., et al. 2007, A\&A, 474, 105

Caloi, V., \& D’Antona, F. 2005, A\&A, 463, 987

Caloi, V., \& D'Antona, F. 2007, A\&A, 463, 949

Canuto, V. M. C., \& Mazzitelli, I. 1991, ApJ, 370, 295

Carretta, E. 2006, AJ, 131, 1766
Carretta, E., Bragaglia, A., Gratton, R. G., et al. 2006, A\&A, 450, 523

Cavallo, R. M., Sweigart, A. V., \& Bell, R. A. 1998, ApJ, 492, 575

Cloutman, L., \& Eoll, J. G. 1976, ApJ, 206, 548

Cohen, J. G., Briley, M. M., \& Stetson, P. B. 2005, AJ, 130, 1177

Cottrell, P. L., \& Da Costa, G. S. 1981, ApJ, 245, L79

D’Antona, F., \& Caloi, V. 2004, ApJ, 611, 871

D'Antona, F., \& Caloi, V. 2008, MNRAS, in press

D’Antona, F., \& Mazzitelli, I. 1996, ApJ, 473, 550

D'Antona, F., \& Ventura, P. 2007, MNRAS, 379, 1431

D'Antona, F., Caloi, V., Montalban, J., Ventura, P., \& Gratton, R. 2002, A\&A, 395,69

D’Antona, F., Bellazzini, M., Caloi, V., et al. 2005, ApJ, 631, 868

Decressin, T., Meynet, G., Charbonnel, C., Prantzos, N., \& Ekström, S. 2007, A\&A, 4641029

Denissenkov, P., \& Herwig, F. 2003, ApJ, 590, L99

Denissenkov, P., \& Weiss, A. 1996, A\&A, 308, 773

Denissenkov, P., \& Weiss, A. 2001, ApJ, 559, L115

Denissenkov, P., Da Costa, G. S., Norris, J. E., \& Weiss, A. 1998, A\&A, 333, 926

D’Ercole, A., Vesperini, E., D’Antona, F., McMillan, S. L. W., \& Recchi, S. 2008, MNRAS, in press [arXiv: 0809.1438]

Eggleton, P. P., Deaborn, D. S. P., \& Lattanzio, J. C. 2008, ApJ, 677, 581

Fenner, Y., Campbell, S., Karakas, A. I., Lattanzio, J. C., \& Gibson, B. K. 2004, MNRAS, 353, 789

Ferguson, J. W., Alexander, D. R., Allard, F., et al. 2005, ApJ, 623, 585

Formicola, A., Imbriani, G., Costantini, H., et al. 2004, Phys. Lett. B, 591, 61

Frost, C. A., Cannon, R. C., Lattanzio, J. C., Wood, P. R., \& Forestini, M. 1998, A\&A, 332, L17

Harris, W. E. 2003, Catalog of parameters for the Milky Way Globular Clusters, http: //www . physics.mcmaster.ca/harris/mwgc.dat

Gratton, R., Bonifacio, P., Bragaglia, A., et al. 2001, A\&A, 369, 87

Gratton, R. G., Bragaglia, A., Carretta, E., et al. 2003, A\&A, 408, 529

Gratton, R., Sneden, C., \& Carretta, E. 2004, ARA\&A, 42, 385

Grevesse, N., \& Sauval, A. J. 1998, SSRv, 85, 161

Herwig, F. 2004, ApJS, 155, 651

Herwig, F. 2005, ARA\&A, 43, 435

Hale, S. E., Champagne, A. E., Iliadis, C., et al. 2002, Phys. Rev. C, 65, 5801

Hale, S. E., Champagne, A. E., Iliadis, C., et al. 2004, Phys. Rev. C, 70, 5802

Iglesias, C. A., \& Rogers, F. J. 1996, ApJ, 464, 943

Ivans, I. I., Sneden, C., Kraft, R. P., et al. 1999, AJ, 118, 1273

Izzard, R. G., Lugaro, M., Karakas, A. I., Iliadis, C., \& van Raai, M. 2007, A\&A, 466,641

Johnson, J. A., Herwig, F., Beers, T. C., \& Christlieb, N. 2007, ApJ, 658, 1203

Karakas, A., \& Lattanzio, J. C. 2007, PASA, 24, 103

Kraft, R. P. 1994, PASP, 106, 553

Kroupa, P., Tout, C. A., \& Gilmore, G. 1993, MNRAS, 262, 545

Lee, Y.-W., Joo, S.-J., Han, S.-I., et al. 2005, ApJ, 621, L57

Lucatello, S., Tsangarides, S., Beers, T. C., et al. 2005, ApJ, 625, 825

Maiolino, R., Oliva, E., Ghinassi, F., et al. 2004, A\&A, 420, 889

Norris, J. E. 2004, ApJ, 612, L25

Piotto, G., Villanova, S., Bedin, L. R., et al. 2005, ApJ, 621, 777

Piotto, G., Bedin, L. R., Anderson, J., et al. 2007, ApJ, 661, L53

Prantzos, A., \& Charbonnel, C. 2006, A\&A, 458, 135

Salpeter, E. E. 1955, ApJ, 121, 161

Saumon, D., Chabrier, G., \& Van Horn, H. M. 1995, ApJS, 99, 713

Smith, V. V., Cunha, K., Ivans, I. I., et al. 2005, ApJ, 633, 392

Sneden, C., Kraft, R. P., Prosser, C. F., \& Langer, G. E. 1991, AJ, 102, 2001

Sneden, C., Kraft, R. P., Shetrone, M. D., et al. 1997, AJ, 114, 1964

Sneden, C., Kraft, R. P., Guhathakurta, P., Peterson, R. C., \& Fulbright, J. P. 2004, AJ, 127, 2162

Stolzmann, W., \& Blöecker, T. 2000, A\&A, 361, 1152

Sweigart, A. V., \& Mengel, J. G. 1979, ApJ, 229, 624

Anthony-Twarog, B. J., \& Twarog, B. A. 2000, AJ, 120, 3111

van Loon, J. T., Zijlstra, A. A., \& Groenewegen, M. A. T. 1999, A\&A, 346, 805

Ventura, P., \& D’Antona, F. 2005a, A\&A, 431, 279

Ventura, P., \& D'Antona, F. 2005b, A\&A, 439, 1075

Ventura, P., \& D'Antona, F. 2006, A\&A, 457, 995

Ventura, P., \& D'Antona, F. 2008a, A\&A, 479, 805

Ventura, P., \& D'Antona, F. 2008b, MNRAS, 385, 2034

Ventura, P., Zeppieri, A., D’Antona, F., \& Mazzitelli, I. 1998, A\&A, 334, 953

Ventura, P., D’Antona, F., \& Mazzitelli, I. 2000, A\&A, 363, 605

Ventura, P., D'Antona, F., Mazzitelli, I., \& Gratton, R. 2001, ApJ, 550, L65

Ventura, P., D’Antona, F., \& Mazzitelli, I. 2002, A\&A, 393, 215

Vitense, E. 1953, Z. Astrophys., 32, 135

Wachter, A., Winters, J. M., Schröder, K.-P., \& Sedlmayr, E. 2008, A\&A, 486, 497 